\title{
Sleep Health and Its Related Influencing Factors in Primary and Middle School Students in Fuzhou: a Large Multi-Center Crowd-based Cross-sectional Study
}

\section{Xingyan Xu}

Fujian Medical University

\section{Fuhao Zheng}

Fujian Provincial Hospital

\section{Yingying Cai}

Fujian Medical University

Jie Lin

Second Affiliated Hospital of Fujian Medical University

\section{Zhaonan Zeng}

Fujian Provincial Hospital

\section{Shichao Wei}

Fujian Provincial Hospital

Siying Wu ( $\sim$ fmuwsy@163.com )

Fujian Medical University

\section{Research article}

Keywords: Sleep health, Children and adolescents, Factor, Fuzhou

Posted Date: September 30th, 2020

DOl: https://doi.org/10.21203/rs.3.rs-77050/v1

License: (c) (i) This work is licensed under a Creative Commons Attribution 4.0 International License. Read Full License 


\section{Abstract}

\section{Background}

This current study set out to investigate the status of sleep health in 7-20-year-old students in Fuzhou and explore the related influencing factors of sleep health.

\section{Methods}

A total of 38467 children and adolescents in Fuzhou were included in the study through a random stratified cluster sampling. Data were collected from May-June 2019, in 18 primary schools and 18 middle schools from 9 Districts, Fuzhou. Children's parents and adolescents of sampled classes were invited to fill a series of questionnaires about the performance of the last 6 months (sociodemographic characteristics, family sleep habits and routine activities before bedtime). Multiple linear regression was carried out to analyze data.

\section{Results}

Of the total 40888 questionnaires we released, 38467 were valid and effective with the response rate was $94.08 \%$. The age of the surveyed participants was $11.85 \pm 3.1$, including 20013 boys and 18454 girls. The multiple linear regression analysis identified factors associated with sleep health $(P<0.05)$ : $\mathrm{Grl}$ (coef $=-0.073,95 \% \mathrm{Cl}$ - $-0.115-0.03)$, Age (coeff $=0.234,95 \% \mathrm{Cl}$. 0.224-0.243), Key school (coeff $=-0.149$, 95\% Cl: -0.193- -0.105), Urban (coeff $=-0.096,95 \% \mathrm{Cl}$ : -0.139-0.054), Excessive daytime sleepiness (coeff $=0.535,95 \%$ Cl: 0.432-0.639), Unhealthy sleep habits (coeff $=0.363,95 \%$ Cl: 0.307-0.419), Using electronic products in bedroom (coeff $=0.074,95 \% \mathrm{Cl}$. 0.028-0.121), Screen time per day during school (coeff $=0.260,95 \% \mathrm{Cl}: 0.235-0.284$ ), Frequency of using electronics 30 minutes before bedtime (coeff $=0.150,95 \% \mathrm{Cl}$ : 0.134-0.166), Eating before sleep (coeff $=0.578,95 \% \mathrm{Cl}$ : 0.527-0.63), Sleep alone (coeff $=-0.204,95 \% \mathrm{Cl}$ : $-0.262-0.147)$ Intense relationship with parents (coeff $=0.361,95 \% \mathrm{Cl}$ : 0.27-0.452), Intense relationship with peers (coeff $=0.267,95 \% \mathrm{Cl}$ : 0.171-0.363), Excessive homework or learning (coeff $=0.189,95 \% \mathrm{Cl}$ : 0.141-0.237), Time for doing homework (coeff $=0.266,95 \% \mathrm{Cl}$. $0.245-0.287$ ) and Mood swings frequently (coeff $=1.174,95 \%$ Cl. 1.127-1.221) had greater impact on sleep health than other factors.

\section{Conclusions}

Sleep health is associated with factors covered sociodemographic characteristics, family sleep habits and routine activities before bedtime, and multiple measures should be taken to improve sleep quality in a targeted manner.

\section{Background}

Sleep health is a notable concern during school age and adolescence, with the majority of them reporting poor sleep patterns, including insufficient and inferior quality sleep. Sleep problems are estimated to 
affect $30-70 \%[1]$ of adolescents in Europe and North America and 14\% to $68 \%$ of students sleeping less than recommended on school days[2]. Just half $(50.19 \%)$ of the children slept for $>8 \mathrm{~h}$ per days and only one-third of children (35.37\%) reported good sleep quality in China[3]. Compared with other age groups, healthy sleep is challenged by unique features for elementary and junior high school students: extrinsic factors such as social activities and academic demands (with early school start times) interact with autonomy regarding their sleep schedules that cause irregular sleep patterns[1]. Previous research has shown that poor sleep in children and adolescents to be associated with a variety problematic outcomes including reduced life satisfaction[4], increased feelings of anxiety and depression[5], increased diagnosis of diseases (attention-deficit/ hyperactivity disorder[6], obesity[7], and diabetes[8], and increased risk for suicide[9], underscoring the need to include healthy sleep into students health promotion efforts.

Given the high prevalence of sleep deficiency in children and adolescents, the negative impact on a range of outcomes, and the particular sleep characteristics, it is important to assess factors influencing sleep health in this age group for improve their sleep quality. Sleep health is multifaceted, and requires adequate duration, high quality and without disturbance such as difficulty falling and staying asleep[10]. However, only a few studies have been conducted on sleep health of primary and middle school students, and even most of these studies focused on sleep duration with little attention to sleep quality. Besides, comparable data about sleep deficiency in children and adolescents from China is limited and related research in Fujian Province is scarce based on interview literatures. Hence, this study will therefore provide such estimates.

In this study, data on sleep collected in school-based surveys of primary and middle school students in Fuzhou, Fujian Province, China, were enrolled to describe the sleep patterns in children and adolescents and to assess the factors influencing sleep health (involving sleep quality, sleep duration and sleep disturbance). This is a rare large-scale study on the sleep health of primary and middle school students in China, which fills the gap of relevant studies in Fujian Province and provides a theoretical basis for formulating measures to improve the sleep of children and adolescents.

\section{Methods}

A cross-sectional, randomized, stratified, multistage cluster sampling methodology was conducted to select 36 schools of Fuzhou, a city divided into 13 districts classified as 3 regions including 6 urban Districts, 6 ruralDistricts and 1 County-level city. Based on the statistical formula:

$$
N=\operatorname{deff} * \frac{\left(Z_{\alpha / 2}\right)^{2} * P^{*}(1-P)}{\delta^{2}}
$$

(where $n, p$, and $\delta$ are sample size, positive rate, and acceptable error, respectively), supposing sleep disorder prevalence $=4.43 \%$ in children[11], significance at $a=0.05$ with $Z_{a / 2}$ of 1.96 , and acceptable error at $0.1 \mathrm{P}$ the sample size was calculated as 8288 . Allowing for nonresponse (deff $=2)$, the final intended sample size was set as 16576. In stage 1, 5 urban Districts and 4 rural Districts were randomly selected from all 13 Districts (7 urban and 6 rural) in Fuzhou. In stage 2, 2 primary schools and 2 junior middle 
schools were randomly chosen from the selected Districts (Taijiang District, Mawei District, Canshan District, Fuqing District, Minhou District, Minqing District, Yongtai District, Gulou District and Jinan District). In stage 3 , classes were selected by the principals in reasoned consideration of the class schedule. Participation was voluntary and anonymous. All students beside Grade 6, 9 and 12 in chosen schools were invited to participate in the survey. The students in the presence of a researcher and children's parents completed the self-administered questionnaire about the performance of the last 6 months in normal classroom settings. Data collection occurred from May to June in 2019.

\section{Questionnaire investigation}

The sleep quality assessment uses a sleep self-made questionnaire, which is divided into three dimensions: sleep duration, sleep disturbance, and subjective sleep quality (Table S1). The sum of these three dimensions is sleep health score. The higher the score was got, the worse the sleep health became.

Sleep duration involves 4 items (What time do you go to bed on weekdays and weekends? How many hours do you sleep on weekdays and weekends?). Sleep disturbance includes 6 items (Do you have nightmares while sleeping? Is there any apnea or awakening while sleeping? Will you wake up more than 2 times a night? Is it difficult to fall asleep during waking up at night? Do you often feel that you can't move when you wake up? Do you feel physical pain during sleep?). Subjective sleep quality dose consist of 3 items (Did you have a good sleep? How long have you been sleepless? Do you feel lack of sleep?).

Reliability tests are tests aiming at improving the precision of a questionnaire. Cronbach's alpha coefficient (a) is used to evaluate the internal consistency. In our survey, Cronbach's a of the present study indicated good reliability of the scale (Cronbach's $a=0.716$ ). Pearson correlation coefficients is used to assess the test-retest reliability. The correlation between overall sleep quality and three dimensions and the distinguishing validity of the scale. Contract validity is measured by Principal components analysis. After standardizing the scores of all items of each scale and establishing a correlation coefficient matrix, Principal components analysis is used to verify Contract validity, extracting the common factor through the fourth-order maximum orthogonal rotation. See appendix for the details (Table S2 and S3).

In addition, questionnaires were dealt with demographic characteristics (sex, age, type of school, area), lifestyle behaviors [Excessive daytime sleepiness, Unhealthy sleep habits, Unhealthy eating habits, Eating before sleep, electronic-products usage (Using electronic products in bedroom, Screen time per day during school, Frequency of using electronics 30 minutes before bedtime) and Sleep alone], emotional factors [Intense relationship with parents, Intense relationship with peers or heavy academic stress (Excessive homework or learning, Time for doing homework) and Mood swings frequently].

\section{Statistical analysis}

Data collation and analysis were conducted using Epidata 3.0 and SPSS 19.0. Descriptive analysis was performed. Results for continuous variables with normal distributions were described by mean \pm standard deviations (SD) and compared using two-tailed Student's t-test. Results for discrete variables were 
displayed as percentage and distribution differences were examined by the chi square $(\chi 2)$ test. We estimated the strength of the association between overall sleep quality score and predictors by regression coefficient (coef) and $95 \%$ confidence interval $(\mathrm{Cl})$. Two-sided $P<0.05$ was considered to statistical significance.

\section{Results}

In total 40888 questionnaires we released, 38467 completed questionnaires were collected. Thus, the valid response rate was $94.08 \%$. The age of the surveyed participants was $11.85 \pm 3.1$, including 20013 (52\%) boys and 18454 (48\%) girls. Demographic and background characteristics of participants were shown in Table 1. Among of all subjects in the sample in this study, $20013(52.0 \%)$ were male. Almost two-thirds of the participants (62.0\%) were from key schools. In terms of area, $47.3 \%$ lived in urban while $52.7 \%$ lived in rural. Mean age was $11.9 \pm 3.1$ years, and the distribution of age was $53.0 \%$ for $\leq 12$ years, $30.6 \%$ for $13-15$ years and $16.3 \%$ for $\geq 16$ years (Table 1 ).

Table 2 showed the results from comparison of sleep scores by different characteristics. In participants aged $\leq 12$ years, boys and students who studied in urban had a higher sleep health score as well as sleep duration score, sleep disturbance score and subjective sleep quality score but interestingly, this was the opposite of participants aged $>12$ years. In terms of types of school, children and adolescents in key schools scored higher on sleep health compared with others.

We investigated the relationship between behavioral and emotional factors and sleep quality by comparing the sleep health score, sleep duration score, sleep disturbance score and subjective sleep quality score of children according to presence or absence of behavioral and emotional problems (Table 3 and Figure 1). We found that behavioral factors such as Excessive daytime sleepiness, Unhealthy sleep habits, Unhealthy eating habits, Eating before sleep, electronic-media use (Using electronic products in bedroom, Screen time per day during school, Frequency of using electronics 30 minutes before bedtime) and Sleep alone cause a unhealthy impact in sleep quality. In terms of emotional factors, participants who suffered from strained relations with parents/peers or heavy academic stress (Excessive homework or learning, Time for doing homework) had poor sleep quality. And moreover, sleep health score was significantly higher in the presence of fluctuating emotions.

Additionally, we also found that the correlation coefficient ( $r$ ) between any two factors was statistically significant $(P \otimes 0.05)$. Sleep health was positively correlated with sleep duration $(r=-0.72)$, sleep disturbance $(r=0.73)$, subjective sleep quality ( $r=0.8)$ (Figure 1 ).

A: sleep health; B: sleep duration; C: sleep disturbance; D: subjective sleep quality, E: time for doing homework, F: Screen time per day during school , G: Frequency of using electronics 30 minutes before

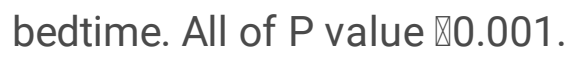

A multiple linear regression was conducted to determine the influence of lifestyle and other factors on sleep health using "Backward elimination". We including the significant factors in above Student's t-test 
and demographic characteristics into the equation of a multiple linear regression, it shows items such as demographic characteristics (sex, age, type of school, area), lifestyle behaviors [Excessive daytime sleepiness, Unhealthy sleep habits, Unhealthy eating habits, Eating before sleep, electronic-products usage (Using electronic products in bedroom, Screen time per day during school, Frequency of using electronics 30 minutes before bedtime) and Sleep alone], emotional factors [Intense relationship with parents, Intense relationship with peers or heavy academic stress (Excessive homework or learning, Time for doing homework) and Mood swings frequently] that affected sleep health. Furthermore, Mood swings frequently was considered the most influential factor on overall variables (Table 4).

\section{Discussion}

Healthy sleep is essential for children's and adolescents' physical and mental well-being. As we know, the investigation of sleep problems for children and adolescents are relatively limited in China up to now. In the present study conducted in a large sample of elementary and junior high school students in Fujian Province, China, we explore whether sleep health (sleep duration, sleep disturbance, subjective sleep quality) was associated with behavioral, social and physical factors to provide some reference value for suggestions for their sleep health in the future.

We observed sex, age, types of school and region differences that suggest need for tailored sleep promotion recommendations for children and adolescents with different characteristics. Boys had a higher sleep health scores and had lower sleep quality than girls, which is in the line with that of previous studies[12,13]. The older the age in our study, the higher the sleep score, where a higher score means poorer sleep. Some biological factors such as reduced homeostatic sleep pressure and delayed circadian rhythms in conjunction, as well as early high school start times and psychological changes from childhood to adolescence, might account for the differences in sleep among children and adolescents[14,15]. Elementary and junior high school students living in urban areas had more adverse sleep than those living in rural areas. Urban students have more night activities compared to rural students because of social and economic developmental disparities, which might result in reduced sleep duration of urban students and have a negative impact on sleep[16]. The types of school difference in children and adolescents is also indicated in this study. A 12-country study demonstrates that experiencing some or a lot of fast-paced and highly competitive school environment (in comparison to none or a little school pressure) was associated with a decrease of sleep duration of 4.56 min per day and an increase of sleep onset difficulties of 0.32 respectively[17]. Based on this study as well as previous researches, we speculate that the difference may be due to students in key schools suffer from a high academic burden and test stress, placing them under a high level of psychological stress that may aggravate the sensitization of the sleep system and lead to heightened alertness, physiological arousal and difficulty falling asleep[18].

It is known lifestyles have an effect on sleep health. In the current study, unhealthy living and behavioral characteristics including Excessive daytime sleepiness, Unhealthy sleep habits, Eating before sleep, social media usage (Screen time per day during school, Frequency of using electronics 30 minutes before 
bedtime, Using electronic products in bedroom) and Sleep alone have caused negative influence on sleep health.

Our study result shows that activities related to electronic products or media exposure before bedtime increased the incidence of sleep problem in children and teenagers and found one in five adolescents or children were classed as very high users, the crowd of using social media before bedtime everyday. With the popularity of electronic products or media, the proportion of users who own mobile phones increased, which has caused a series of critical health problems[19,20,21]. Accumulating studies for the past 10 years suggested that students who had using electronic products or media habit before bedtime had made easily sleep health worse than others[12,22,23]. Consistent with these studies, Akçay D, et al. demonstrated that as the teenager in Konya High School spent more time on their social media, their sleep quality become progressively worse[24]. The decrease of self-reported sleep duration among U.S. adolescents 2009-2015 correlated with new media screen time[25]. More than 50\% of adolescents in our study had electronic products or media such as computer, iPad, callphone or television in their bedroom. Continente $\mathrm{X}$, et al. demonstrated that adolescents with media devices in their bedroom were more likely to be short sleepers[26]. Through electronic products or media (computer, Internet, smartphone, et al) usage, teenagers often play games, watch movies, TV-series, or entertainment shows, chat online or other activities. These activities, a stimulus for brain stimulating neurons contributed to increasing the occurrence adverse sleep outcomes in students. Moreover, as long time use of screen at night, eyes of students are exposed to strong light, and its blue spectrum has a strong and specific effect on the retina[27]. Light can not only prevent the secretion of melatonin to leading to circadian rhythm disorder, but also cause the desire of awakening to delaying sleep[28]. Besides, some adolescents reported have difficulties disengaging from social media to sleep[29].

$19.9 \%$ of the current sample had co-sleeper, which associate negatively with sleep health. Due to cosleepers may do more activities, such as talking and playing before going to bed, or made sleeping environment noisy, sleep problems are prone to come out easily in students sharing bed or room[30], which makes it easier for students to fall into the unhealthy habit of sleep. Meanwhile, our results also shows unhealthy sleep habits such as getting up late in the morning, stay up late, and energetic exercise before bed, was related with sleep health. Furthermore, excessive daytime sleepiness are related significantly to sleep health. We suspect if a student is drowsy during the day, there will be fragmentation of night sleep and increased sleep pressure, which will affect night sleep. Our findings suggest that food intake preceding sleep have a negative impact on sleep health, consistent with previous studies[31,32]. A previous study showed snacking and drinking caffeinated beverages before going to bed were associated with significantly short sleep duration and poorer sleep quality ( $\mathrm{OR}=1.49$ and 1.83 , respectively)[33].

Relationship with families and peers also appeared to play a pivotal role in sleep health. Because home and school are the two places which students' lives mainly involve in, students who received more social support from family and friends reported greater life satisfaction. According to previous research, for children and adolescents, higher psychosocial well-being was linked to longer nocturnal sleep duration and lower levels of sleep disturbances[34]. In our study, students who are satisfied with their family 
life/classmates relationship sleep better. The hypothalamic-pituitary -adrenal axis was activated with the release of hormones affect sleep architecture due to endure stress[35] which in childhood can emerge from various sources like problems with the family and peers[36].

In the current study, fluctuation of emotions an important factor for unhealthy sleep, which is consistent with previous studies[37,38]. Emotion and sleep are closely linked and many studies have shown that heightened emotional reactivities including strong positive and negative emotions are related to the maintenance of insomnia symptoms[39]. According to previous studies, affective processes mediate the effect of cognitive and autonomic hyperarousal on sleep[40]. Sleep problems are confirmed to be associated with decreased levels of vagal suppression, which is considered indices of emotion regulation. Additionally, higher levels of emotional intensity tied in with reduced sleep duration and increased nocturnal activity[41]. However, the relationship between mood and sleep is complex and bidirectional because poor emotions can worsen sleep and vice versa[42], further research is needed.

To data, comparable data on sleep deprivation among children and adolescents in Fujian is limited, but our study adds relevant research evidence in this aspect. Furthermore, another strength in the current study is a Cross sectional study of relatively large sample size and comprehensive assessments on a series of sleep health variables, which make us research on the potential factors influencing sleep. However, several limitations should be noted. The first is that causality will be difficult to demonstrate in cross-sectional design, although there are multiple theories to support our findings. Second, the current research does not include factors such as family structure and socioeconomic status, school timetable and parents' sleeping habits, which may be significantly related to sleep health of Chinese students. Moreover, as a result of graduation having heavy academic stress, the last-year students (grades 6, 9 and 12) were not included into investigate.

\section{Conclusion}

Overall, this study found that a series of factors are significantly associated with adverse sleep outcomes among children and adolescents, China. The results of study have valuable clinical significance as our findings indicated that the existing sleep problem in children and teenager could be, at least partly, intervened by reducing the use of electronic products before bedtime, especially cellphone, by avoiding mood swings frequently and by improving the routine habit before bedtime. Based on our results, we recommend the combination of school with family intervention, and parents, teachers, health professionals and students should raise awareness of developing healthy sleep habits in usual life, which may be more effective in improving students sleep health.

\section{Abbreviations}

Cl: Confidence interval; OR: Odds ratio

\section{Declarations}




\section{Ethics approval and consent to participate}

The survey was approved by the Ethics Committee of Fujian Medical University. According to the voluntary principle, a parent or guardian for participants under 16 years old and the respondentsor over 16 year old can agree to and sign informed consent and suspend participation at any time in the study .

\section{Consent for publication}

Not applicable.

\section{Availability of data and materials}

The datasets generated and/or analysed during the current study are not publicly available due the principle of confidentiality of funding but are available from the corresponding author on reasonable request.

\section{Competing interests}

The authors declare no conflict of interest.

\section{Funding}

This research was funded by Joint Funds for the Innovation of Science and Technology, Fujian Province (2018Y9089, received by Siying Wu), the Natural Science Foundation of Fujian Province (2019J01315, received by Siying Wu), Professor Development Fund Project of Fujian Medical University (JS15002, received by Siying $\mathrm{Wu}$ ), Investigation and comprehensive exploration of intervention strategies on obesity and nutritional status of primary and middle school students in Fuzhou (2019B011, received by Siying $\mathrm{Wu}$ ) and Fujian Provincial hospital-high level hospital construction program (2018-GSP-001, received by Shichao Wei), which supported data analysis, quality control and English editing).

\section{Author Contributions}

Designed and modified the manuscript: SCW and SYW; Designed the research and participated in the experimental design, coordinated and drafted the manuscript: $X X, F Z, Y C, J L, Z Z$; Data collection, achievement interpretation and manuscript writing: XX, FZ, YC, JL, ZZ; Analysed the data: XX, YC. All authors have read and agreed to the published version of the manuscript.

\section{Acknowledgements}

We are particularly grateful for the willingness of our participants to participate in our study the participants and participating physicians for their participation in this study. We thank the staffs of Fujian Provincial Hospital, China for making this research possible.

\section{Authors' information}


1 Department of Epidemiology and Health Statistics, School of Public Health, Fujian Medical University, Minhou County, Fuzhou, Fujian, China

2 Office of Academic Research, Fujian Provincial Hospital, Fuzhou 350001, China

3 The Second Attached Hospital of Fujian Medical University, Quanzhou 362000, China

${ }^{4}$ Center for Experimental Research in Clinical Medicine, Fujian Provincial Hospital, Fuzhou 350001, China

${ }^{5}$ Sleep medicine center, Fujian Provincial Hospital, Fuzhou 350001, China

\section{References}

1. Michael G, Greg G, Hayley D. Recent worldwide sleep patterns and problems during adolescence: a review and meta-analysis of age, region, and sleep. Sleep Med, 2011, 12: 110-118.

2. Genevieve G, Sofia D, Inese G, Mette R, Margarida G.M., Jorma T, lan J, Michal K, Anita V, Daniela H, Fiona B, Frank J.E., Solvita K.M., Kastytis Š, Tania G, Christina S. How Are Adolescents Sleeping? Adolescent Sleep Patterns and Sociodemographic Differences in 24 European and North American Countries. J Adolesc Health, 2020, 66: S81-S88.

3. Xu T, Liu J, Zhu G, Han S. Prevalence and Associated Lifestyle Factors of Suboptimal Health Status among Chinese Children Using a Multi-Level Model. Int J Environ Res Public Health, 2020, 17(5): 1497.

4. Courtney K.B., Lauren E.H., Amy J.E., Christopher B.F., Jody G, Kelly J.H., Carlos A.C.Jr., Monique K.L, program collaborators for Environmental influences on Child Health Outcomes (ECHO). Better sleep, better life? How sleep quality influences children's life satisfaction. Qual Life Res, 2020.

5. Faith O, Alice M.G., Michael G, Shirley R. Self-reported sleep patterns and quality amongst adolescents: cross-sectional and prospective associations with anxiety and depression. J Child Psychol Psychiatry, 2020 (Online ahead of print).

6. Liu X, Liu Z, Liu P, Sun S, Jia Associations between sleep problems and ADHD symptoms among adolescents: findings from the Shandong Adolescent Behavior and Health Cohort (SABHC). Sleep, 2020, 43(6): zsz294.

7. Chelsea L.K., Jean-Philippe C, Corby K.M., Catherine M.C., Peter T.K, Amanda E.S. Associations of Sleep with Food Cravings, Diet, and Obesity in Adolescence. Nutrients, 2019, 11(12): 2899.

8. Julia von S, Claudia B, Stephanie B, Beate K, Desiree D, Angela G, Christian D, Friederike D, Heike V, Martin W, Till R, Celine V. Sleep and glycemic control in adolescents with type 1 diabetes. Pediatr Diabetes, 2018, 19: 143-149.

9. Eren Y, Rukiye S, Ümit I, Mustafa S.Ö, Medine A.T., ilyas C, Bulent A. Comparison of the Sleep Problems Among Adolescents Who Attempted Suicide and Healthy Adolescents. Nerv Ment Dis, 2020, 208: 294-298.

10. Buysse D.J.. Sleep health: can we define it? Does it matter? Sleep, 2014, 37: 9-17. 
11. Huang M, Qian Z, Wang J, Michael G.V, Lee L.Y., Dong G. Validation of the sleep disturbance scale for children and prevalence of parent-reported sleep disorder symptoms in Chinese children. Sleep Med, 2014, 15: 923-928.

12. Li X, Tai J, Xu Z, Wang G, Wu Y, Du J, Zhang J, Peng X, Ni Sleep Duration and Factors Related to Sleep Loss in 3-14-Year-Old Children in Beijing: A Cross-Sectional Survey. Chin. Med J, 2018, 131: 1799-1807.

13. Sarah J, Anne-Marie C, Orfeu M.B., Lauren H. Disparities in adolescent sleep health by sex and ethnoracial group. SSM Popul Health, 2020, 11: 100581.

14. Barclay N.L., Gregory A.M.. Sleep in childhood and adolescence: age-specific sleep characteristics, common sleep disturbances and associated difficulties. Curr Top Behav Neurosci, 2014, 16: 337-365.

15. Palmer C.A.. Tired Teens: Sleep Disturbances and Heightened Vulnerability for Mental Health Difficulties. J Adolesc Health, 2020, 66: 520-521.

16. Jinseok K, Jin-Won N, Ahraemi K, Young D.K.. Demographic and Socioeconomic Influences on Sleep Patterns among Adolescent Students. Int J Environ Res Public Health, 2020, 17(12):

17. Jinseok K, Jin-Won N, Ahraemi K, Young D.K.. Does Sleep Mediate the Association between School Pressure, Physical Activity, Screen Time, and Psychological Symptoms in Early Adolescents? A 12Country Study. Int J Environ Res Public Health, 2019, 17(12):

18. David A.K., Jason R.A., Christopher L.D.. The impact of stress on sleep: Pathogenic sleep reactivity as a vulnerability to insomnia and circadian disorders. J Sleep Res, 2018, 27: e12710.

19. Holly S, Stephany M.B., Heather C.W.. Social media use and adolescent sleep patterns: crosssectional findings from the UK millennium cohort study. BMJ Open, 2019, 9(9): e031161.

20. Erika F, Valter S, Michelle C, Marcos V.R., Thiago M.F., Francisco R.N., Zila M.S, Pamela J.S., Silvia S.M., Sheila C.C.. Excessive Screen Media Use in Preschoolers Is Associated with Poor Motor Skills. Cyberpsychol Behav Soc Netw, 2020, 23: 418-425.

21. Annemarie R.K., Adam M.L., Tess B.C., Mariel S.B., Liu F, Jennifer B.U., Kira R, Junhan C, Matthew G.K., Rob S.M., Jessica L.B.. Digital media use and subsequent cannabis and tobacco product use initiation among adolescents. Drug Alcohol Depend, 2020, 212: 108017.

22. Royant-Parola S, Londe V, Tréhout S, Hartley S. The use of social media modifies teenagers' sleeprelated behavior. Encephale, 2018, 44: 321-328.

23. Xie J, Daphne S.K.C., Alice Y.L., Oi Ching Bernice L.N., Karry M.L., Angela Y.L., Sau Mui A.T., Cindy Sin U.L., Alex M.A.. Relationships Between Television Viewing, Computer and Mobile Phone Usage and Sleep Quality in a Chinese Population: A Community-Based Cross-Sectional Study. Medical Internet Research, 2020: 18095.

24. Akçay D, Akçay B.D.. The influence of media on the sleep quality in adolescents. Turk J Pediatr, 2018, 60: 255-263.

25. Twenge J.M., Krizan Z, Hisler G. Decreases in self-reported sleep duration among U.S. adolescents 2009-2015 and association with new media screen time. Sleep Med, 2017, 39: 47-53. 
26. Xavier C, Anna P, Albert E, Maria J.L.. Media devices, family relationships and sleep patterns among adolescents in an urban area. Sleep Med, 2017, 32: 28-35.

27. Behar-Cohen F, Martinsons C, F. Viénot, Zissis G, Barlier-Salsi A, Cesarini J.P., Enouf O, Garcia M, Picaud S, Attia Light-emitting diodes (LED) for domestic lighting: Any risks for the eye?. Prog Retin Eye Res, 2011, 30(4): 239-257.

28. Christian C, Sylvia F, Doreen A, Jakub S, Matthias B, Achim P, Ralph M, Anna W.J., Oliver S. Evening exposure to a light-emitting diodes (LED)-backlit computer screen affects circadian physiology and cognitive performance. J Appl Physiol, 2011, 110: 1432-1438.

29. Scott H, Woods H.C.. Fear of missing out and sleep: Cognitive behavioural factors in adolescents' nighttime social media use. J Adolesc, 2018, 68: 61-65.

30. Cara A.P, Michelle A.C., Jessica M.M., Candice A.A.. Co-Sleeping among School-Aged Anxious and Non-Anxious Children: Associations with Sleep Variability and Timing. J Abnorm Child Psychol, 2018, 46: 1321-1332.

31. Mohammad K.A.K., Erin L.F., Yen L.C., John P.E, Kate E.S, Paul J. Is it nutrients, food items, diet quality or eating behaviours that are responsible for the association of children's diet with sleep? J Sleep Res, 2017, 26: 468-476.

32. Cibele A.C., Ioná Z.Z., Bruno G.R., Rafael M.D., Sérgio T, Marco T.M.. Relationship between food intake and sleep pattern in healthy individuals. J Clin Sleep Med, 2011, 7: 659-64.

33. Galland B C, de Wilde T, Taylor R W, Smith Sleep and pre-bedtime activities in New Zealand adolescents: differences by ethnicity. Sleep Health, 2020, 6: 23-31.

34. Barbara F.T., Claudia B, Nathalie M, Toomas V, Antonia S, Lucia R, Luis A.M, Fabio L, Jaakko K, Monica H, Regina F, Wencke G, Stefaan D.H., Wolfgang A., IDEFICS and I.Family consortia. Crosssectional and longitudinal associations between psychosocial well-being and sleep in European children and adolescents. J Sleep Res, 2019, 28: e12783.

35. Nollet M, Wisden W, Franks N.P., Sleep deprivation and stress: a reciprocal relationship. Interface Focus, 2020, 10: 20190092.

36. Ryan-Wenger N.A., Sharrer V.W., Campbell K.K.. Changes in children's stressors over the past 30 years. Pediatr Nurs, 2005, 31: 282-8, 291.

37. Jojanneke A.M.C van K., Maurice-Stam H, Antoinette Y.N.S, Dannis G van V, Bernd G, Corrie G, Marieke A.R., Raphaële R.L van L, Martha A. High occurrence of sleep problems in survivors of a childhood brain tumor with neurocognitive complaints: The association with psychosocial and behavioral executive functioning. Pediatr Blood Cancer, 2019, 66: e27947.

38. Emanuela M, Guido A, Davide D, Corrado F, Maurizio F, Cristiano V, Maria A.S.. Adolescents selfreported sleep quality and emotional regulation: a discordant twin study. Ann Ist Super Sanita, 2019, 55: 118-123.

39. Chiara Ba, Kai S, Caterina L, Dieter R. Sleep and emotions: a focus on insomnia. Sleep Med Rev, 2010, 14: 227-38. 
40. Espie C.A.. Insomnia: conceptual issues in the development, persistence, and treatment of sleep disorder in adults. Annu Rev Psychol, 2002, 53: 215-243.

41. El-Sheikh M, Buckhalt J. Vagal regulation and emotional intensity predict children's sleep problems. Dev Psychobiol, 2005, 46: 307-317.

42. Gruber R, Cassoff J. The interplay between sleep and emotion regulation: conceptual framework empirical evidence and future directions. Curr Psychiatry Rep, 2014, 16: 500.

\section{Tables}

Due to technical limitations, table 1, 2, 3 and 4 is only available as a download in the Supplemental Files section.

\section{Figures}




\begin{tabular}{|c|c|c|c|c|c|c|}
\hline A & & & & & & \\
\hline 0.72 & B & & & & & \\
\hline 0.73 & 0.28 & C & & & & \\
\hline 0.8 & 0.41 & 0.33 & D & & & \\
\hline 0.24 & 0.27 & 0.08 & 0.19 & $\mathrm{E}$ & & \\
\hline 0.3 & 0.33 & 0.17 & 0.19 & 0.04 & $\mathrm{~F}$ & \\
\hline 0.4 & 0.45 & 0.23 & 0.24 & 0.13 & 0.52 & G \\
\hline
\end{tabular}

Figure 1

Correlation analysis of factors affecting sleep sleep health.

\section{Supplementary Files}

This is a list of supplementary files associated with this preprint. Click to download.

- tables1to4.docx 